\title{
Pineal Region Gliomas: A Systematic Review of Clinical Features and Treatment Outcomes
}

\author{
OTHMAN BIN ALAMER ${ }^{1,2}$, PAOLO PALMISCIANO ${ }^{3}$, SCOTT E. ROWE ${ }^{4}$, ADITYA DUTTA GUPTA $^{5}$, \\ MARYAM HAIDER ${ }^{6}$, MOHAMMED ALDUHAYMI $^{1}$, AARON A. COHEN-GADOL $^{7}$, \\ KENNY YU ${ }^{8}$, TAREK Y. EL AHMADIEH ${ }^{8}$ and ALI S. HAIDER ${ }^{5,9}$ \\ ${ }^{1}$ College of Medicine, King Saud bin Abdulaziz University for Health Sciences, Riyadh, Kingdom of Saudi Arabia; \\ ${ }^{2}$ King Abdullah International Medical Research Center, Riyadh, Kingdom of Saudi Arabia; \\ ${ }^{3}$ Department of Neurosurgery, Trauma Center, Gamma Knife Center, Cannizzaro Hospital, Catania, Italy; \\ ${ }^{4}$ Nova Southeastern University College of Osteopathic Medicine, Fort Lauderdale, FL, U.S.A.; \\ ${ }^{5}$ Texas A\&M University College of Medicine, Houston, TX, U.S.A.; \\ ${ }^{6}$ Department of Radiology, Baylor College of Medicine, Houston, TX, U.S.A.; \\ ${ }^{7}$ Department of Neurological Surgery, Indiana University School of Medicine, Indianapolis, IN, U.S.A.; \\ ${ }^{8}$ Department of Neurosurgery, Memorial Sloan Kettering Cancer Center, New York, NY, U.S.A.; \\ ${ }^{9}$ Department of Neurosurgery, The University of Texas M.D. Anderson Cancer Center, Houston, TX, U.S.A.
}

\begin{abstract}
Background/Aim: To review the current literature on pineal region gliomas, summarizing the clinical characteristics and treatment outcomes. Materials and Methods: PubMed, Scopus, and Cochrane databases were used to identify relevant articles. Comprehensive clinical characteristic review and survival analysis were conducted. Results: Twelve studies describing 81 patients were included. The median age was 39 years (male $=54.3 \%)$. Fifty patients $(61.7 \%)$ had obstructive hydrocephalus requiring cerebrospinal fluid diversion with either ventriculoperitoneal shunt (VPS) (40.0\%) or endoscopic third ventriculostomy (ETV) (24.0\%). Patients who underwent VPS had significant survival benefits compared to ETV $(p<0.05)$. All patients in our review underwent surgery, and gross-total resection $(\geq 98 \%)$ was achieved in $34.6 \%$. The supracerebellar infratentorial approach was the most employed surgical approach (62.3\%). Chemotherapy was administered in
\end{abstract}

Correspondence to: Othman Bin Alamer, MBBS, Department of Stem Cells and Regenerative Medicine, King Abdullah International Medical Research Center, Ali Al Arini, Ar Rimayah, Riyadh 11481, Kingdom of Saudi Arabia. Tel: +96 60114294444, e-mail: Oabinalamer@gmail.com

Key Words: Pineal region glioma, glioma, pineal region tumors, systematic review.

This article is an open access article distributed under the terms an conditions of the Creative Commons Attribution (CC BY-NC-ND) 4.0 international license (https://creativecommons.org/licenses/by-nc-nd/4.0).
$32.1 \%$ of cases, and radiotherapy in $40.7 \%$. The median overall survival (OS) was 12 months, and the overall one-year survival rate was $60 \%$. Conclusion: This study could not establish a correlation between the extent of tumor resection and positive treatment outcomes. However, among cases with hydrocephalus, patients who underwent VPS placement had better survival as compared to ETV.

Pineal region gliomas are rare neoplasms with variable malignant activity, representing $14-22 \%$ of all pineal region tumors. Typically, they arise from the glial stroma of the pineal gland and its surrounding structures (1). Due to their invasive behavior in an anatomically complex region, pineal region gliomas (PRGs) can be challenging to manage and can have poor outcomes (2). Although survival data on gliomas affecting the Central Nervous System (CNS) have been examined, studies scoping in on survival outcomes of PRGs along with the effects of various relevant therapeutic strategies are largely absent in the literature (3).

As a result of PRGs paucity, a consensus standard of care has not been established, leading to various oncological diagnostic protocols and management strategies that have been practiced without unity (4-7). Current diagnostic workup primarily constitutes radiological modalities, such as MRI, for preliminary diagnosis and subsequent intraoperative histological examination for definitive diagnosis $(8,9)$. In combination with chemoradiotherapy, surgery remains the cornerstone treatment strategy, and several surgical techniques and approaches have evolved to maximize safe tumor resection (10-14). 
Although it is a general assumption that the extent of resection and chemoradiotherapy correlate with survival rate, only a handful of case reports and series have studied these questions $(4,15-17)$. This study reviews the current literature regarding the clinical management of pineal region gliomas, focusing on clinical characteristics, treatment strategies, and survival outcomes.

\section{Materials and Methods}

Literature search. A systematic review was conducted according to the Preferred Reporting Items for Systematic Reviews and MetaAnalyses (PRISMA) guidelines (18). PubMed, Scopus, and Cochrane databases were searched from inception to December 2020. A medical subject headings (MeSH) term and keyword search of each database was conducted using the Boolean operators OR and AND. The terms used were as follows: "pineal" and "glioma."

Study selection. Pre-established inclusion and exclusion criteria were followed. Studies were included with the following criteria: 1) were prospective or retrospective studies involving more than one glioma patient; 2) contained patients aged 18 years or older with histologically confirmed glioma involving the pineal region. Studies were excluded with the following criteria: 1) were meta-analyses, reviews, editorials, letters, or books; 2) contained insufficient clinical data (lacking two of the following: patient demographics, specific histological grading, typing, management and/or outcomes); 3) were not written in English.

Two authors (SR and OBA) independently assessed the titles and abstracts of all extracted papers based on the inclusion and exclusion criteria. Studies that met inclusion criteria were then further evaluated independently with full text review by the same two authors, and disagreements were resolved via a third author (ASH). References of the included articles were also screened to retrieve additional relevant articles.

Data extraction. Data from included studies were extracted by one author (OBA) and confirmed independently by two other authors (ASH and PP) to ensure accuracy. Extraction variables included: authors, date of publication sample size, patients' age and gender, presenting symptoms, histological and radiological features, management course, treatments (i.e., radiotherapy, chemotherapy, or surgical approach), recurrence, and survival. Missing data are either not reported by the author or reported indistinctively with other data that could not be differentiated.

Data synthesis. The primary outcomes of interest were the clinical features, management course, and survival analysis of pineal region gliomas. Meta-analysis was precluded due to heterogeneity in outcome measures and the limited number of studies. For each study, the level of evidence was assessed using the 2011 Oxford Centre For EvidenceBased Medicine guidelines, and the risk of bias was evaluated with the Joanna Briggs Institute (JBI) checklists for case series $(19,20)$.

Statistical analysis. Means and ranges were used to summarize continuous variables, while frequencies and percentages were used to summarize categorical variables. Overall survival (OS) and progression-free survival (PFS) were illustrated using Kaplan-Meier curves. The log-rank test was used to compare survival curves. A two- sided $p$-value $<0.05$ was considered significant for all analyses. Analyses were performed using the statistical software SPSS v. 25 (IBM Corp, Armonk, New York, NY, USA).

\section{Results}

Study selection. The initial literature search yielded 517 citations from PubMed, 654 from Scopus, and 5 from Cochrane (Figure 1). After eliminating duplicates, there were 898 articles. Seven hundred and forty-two studies were excluded based on title and abstract. One hundred and fifty-six full-text articles were then evaluated for inclusion. Of these, 144 papers did not meet inclusion criteria and were subsequently excluded. Twelve articles were included in this systematic review, all categorized as level IV evidence (Table I and Table II) (4, 15, 21-30).

Demographics and clinical characteristics. A total of 81 pineal region gliomas, with slight male predominance $(54.3 \%)$, were included (Table III). Age ranged from 18 to 82 years old, with a median of 39 years. Headache was the main presenting symptom $(20.0 \%)$ followed by decreased visual acuity (16.9\%) and nausea/vomiting (13.8\%). Additionally, 50 patients had hydrocephalus, of whom $64.0 \%$ underwent shunting procedures. The Karnofsky Performance Status (KPS) was reported in 22 patients. The pre-operative and post-operative KPS were equal (KPS $=60 \%$ ), yet postoperative status was reported in only one case $(\mathrm{KPS}=60 \%)$.

The median tumor size was $3.8 \mathrm{~cm}$, ranging from $2 \mathrm{~cm}$ to $6 \mathrm{~cm}$. Of cases with available data, the major histopathological subtypes of glioma included glioblastoma $(n=28 ; 50.0 \%)$, grade II astrocytoma $(\mathrm{n}=11 ; 19.6 \%)$, anaplastic astrocytoma $(n=4 ; 7.1 \%)$, ependymoma $(n=3 ; 5.4 \%)$, gliosarcoma $(n=2$; $3.6 \%)$, pilocytic astrocytoma $(n=1 ; 1.8 \%)$, ganglioglioma $(\mathrm{n}=1 ; 1.8 \%)$, and central neurocytoma $(\mathrm{n}=1 ; 1.8 \%)$, along with 5 astrocytoma cases with unspecified grade (Figure 2). Of all gliomas with available data, $38(67.9 \%)$ were high-grade glioma (HGG - WHO III and IV), whereas 18 (32.1\%) were low-grade glioma (LGG - WHO I and II).

Management strategies and survival outcomes. Recurrence occurred in $12(14.8 \%)$ patients, and death was documented in $70.6 \%$. Median OS was 12 months (range $=2-78$ ), and median PFS was 11.3 months (range=2-78). The one-year OS and PFS rates were $60 \%$ and $51 \%$, respectively (Figure 3); however, glioblastoma notably showed a one-year survival rate of $45 \%$. LGG had better survival than HGG ( $p<0.01$; Figure 4).

Of all cases with hydrocephalus, twenty patients $(40.0 \%)$ had VPS, and 12 (24.0\%) had ETV; however, 18 patients $(36.0 \%)$ did not undergo shunting. In addition, our results suggest a survival benefit for patients who underwent VPS compared to ETV ( $p<0.05$; Figure 5).

All patients in our cohort underwent surgery, of which 14 (17.3\%) underwent biopsy alone. Gross-total resection (GTR; 


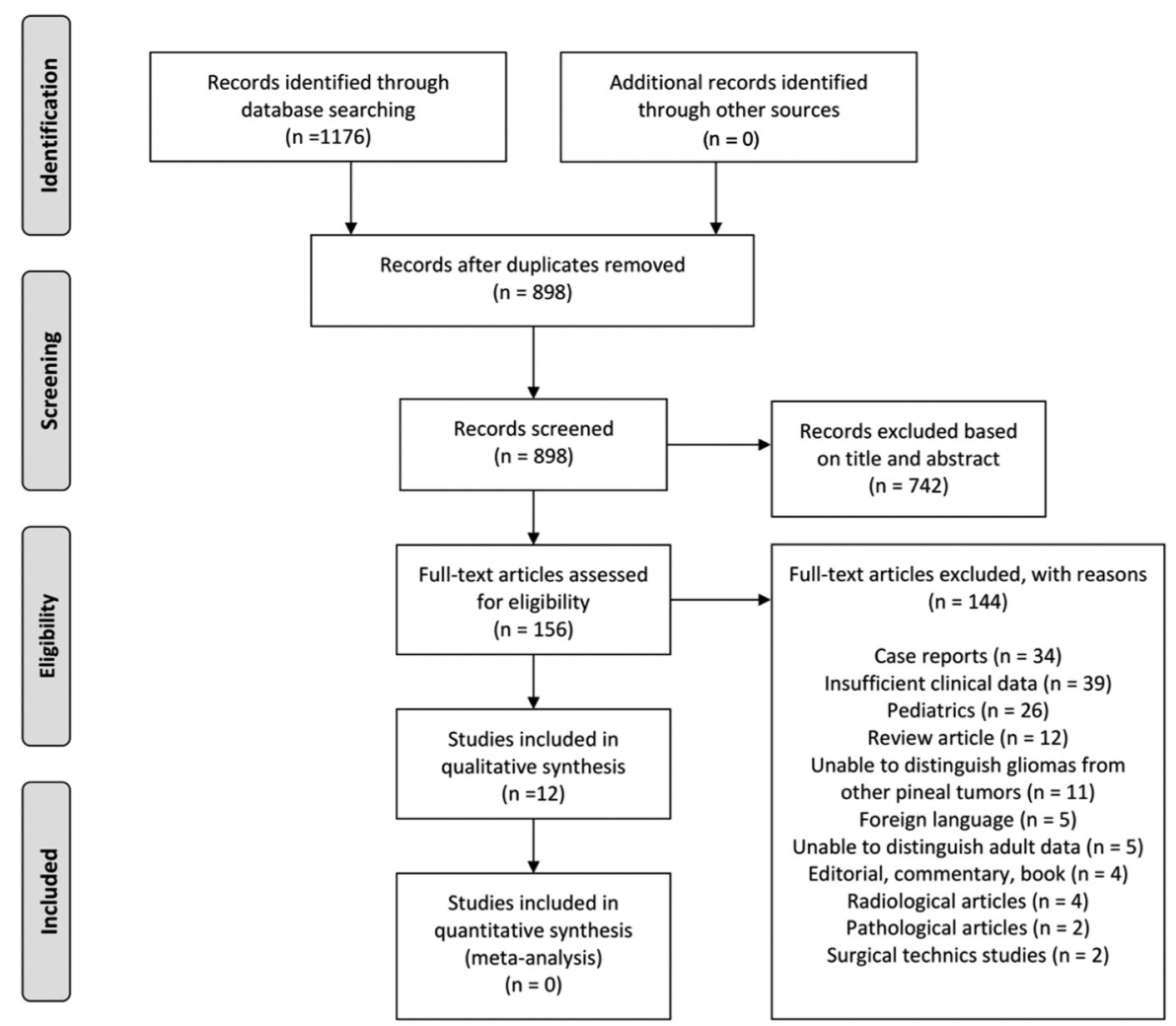

Figure 1. PRISMA flowchart illustrating the search strategy and data selection based on the inclusion and exclusion criteria.

removal of $\geq 98 \%$ of tumor) was achieved in only $34.6 \%$ of cases. Subtotal resection (STR; removal of $80-97 \%$ ) and partial resection (removal of $<80 \%$ ) were achieved in $40.7 \%$ and $7.4 \%$ of cases, respectively. The most frequently used surgical approaches were the supracerebellar infratentorial approach (SCIT) $(n=38 ; 62.3 \%)$, followed by the transcortical transventricular approach (TT) $(\mathrm{n}=11 ; 18.0 \%)$ and the transcallosal interhemispheric approach $(\mathrm{TCIH})(\mathrm{n}=8 ; 13.1 \%)$. Neither extent of resection $(p=0.18)$ nor surgical approaches $(p=0.17)$ demonstrated significant survival differences. Fiftythree patients $(65.4 \%)$ received adjuvant therapy. While $32.1 \%$ of patients had chemotherapy, $40.7 \%$ received radiotherapy with a median dose of 5,770 cGy.

\section{Discussion}

This systematic review on PRGs sheds light on the clinical characteristics, treatment strategies, and survival outcomes. Our data support the overall poor prognosis of this rare group of neoplasms. We found no differences in OS based on surgical approach nor extent of resection. However, VPS showed a better survival benefit compared to ETV ( $p<0.05$; Figure 5) in patients presenting with hydrocephalus.
Demographics and clinical characteristics. Our data indicated a male predominance $(54.3 \%)$ and a median age of 39 years at presentation. In contrast, general data on gliomas indicate a 520 year older age of onset (17). We ascribe the early presentation in our cohort to the region's complexity and confined anatomical space, especially in slow-growing subtypes. Clinical manifestations related to increased intracranial pressure presented largely as headaches (20.0\%) with nausea and vomiting (16.9\%), and they were similar to presenting symptoms of other pineal region tumors in adults and pediatric populations $(31,32)$. This is reasonable considering the proximity of the pineal region to the ventricular system, which puts patients at increased risk of obstruction.

Although our analysis failed to demonstrate significant survival benefits of shunting in hydrocephalus compared to cases without shunting, it remains an essential component of the management paradigm for hydrocephalus secondary to pineal region tumors $(33,34)$.

Our results show a variety of reported radiological features among different types of gliomas. While HGG tended to manifest with heterogeneous enhancement in the pineal region and sometimes infiltrated the surrounding structure, LGG presented well-demarcated mass lesions with 
Table I. Overview of the clinical characteristics of all included studies.

\begin{tabular}{|c|c|c|c|c|c|c|c|c|c|c|c|}
\hline & \multirow{2}{*}{$\begin{array}{l}\text { Authors - } \\
\text { Year }\end{array}$} & \multirow{2}{*}{$\begin{array}{l}\text { Level } \\
\text { of } \\
\text { evidence }\end{array}$} & \multirow{2}{*}{$\begin{array}{c}\text { No. of } \\
\text { patients } \\
\text { (Male \%) }\end{array}$} & \multirow{2}{*}{$\begin{array}{l}\text { Age } \\
\text { median } \\
\text { (range) }\end{array}$} & \multirow{2}{*}{$\begin{array}{l}\text { Symptoms } \\
\text { (No. of } \\
\text { patients) }\end{array}$} & \multicolumn{2}{|c|}{$\mathrm{HCP}$} & \multirow{2}{*}{$\begin{array}{l}\text { Median } \\
\text { KPS } \\
\text { PreOp/ } \\
\text { PostOp }\end{array}$} & \multirow{2}{*}{$\begin{array}{c}\text { Median } \\
\text { tumor } \\
\text { size } \\
(\mathrm{cm})\end{array}$} & \multicolumn{2}{|c|}{ Histopathology } \\
\hline & & & & & & $\begin{array}{l}\text { No. } \\
\text { cases }\end{array}$ & $\begin{array}{l}\text { Modality of } \\
\text { shunting }\end{array}$ & & & Type (count) & WHO Grade \\
\hline 1 & $\begin{array}{l}\text { Niu et al., } \\
2020 \text { (23) }\end{array}$ & IV & $3(100 \%)$ & $30(21-55)$ & Headache (1) & 2 & VPS (2) & - & 2.5 & GBM (3) & High-grade (3) \\
\hline 2 & $\begin{array}{l}\text { Li et al., } \\
2019 \text { (4) }\end{array}$ & IV & $21(48 \%)$ & $36(18-54)$ & NM & 13 & $\begin{array}{l}\text { VPS (6) } \\
\text { ETV (3) }\end{array}$ & $60 / \mathrm{NM}$ & 4 & $\begin{array}{c}\text { GBM (5) } \\
\text { Grade II } \\
\text { astrocytoma (4) } \\
\text { Unspecified type } \\
\text { astrocytoma (5) } \\
\text { Ependymoma (3) } \\
\text { Anaplastic } \\
\text { astrocytoma (2) } \\
\text { Central } \\
\text { Neurocytoma (1) } \\
\text { Gangliogliomas (1) }\end{array}$ & $\begin{array}{l}\text { Low-grade (9) } \\
\text { High-grade (7) } \\
\text { Unspecified (5) }\end{array}$ \\
\hline 3 & $\begin{array}{c}\text { Orrego et al., } \\
2017 \text { (24) }\end{array}$ & IV & $4(75 \%)$ & $49(25-56)$ & $\begin{array}{c}\text { N/V (4) } \\
\text { Ataxia (3) } \\
\text { Headache (3) } \\
\text { Dizziness (1) } \\
\text { Vertical gaze } \\
\text { palsy (2) } \\
\text { IV CN palsy (2) } \\
\text { Hemiparesis (1) } \\
\text { Decrease visual } \\
\text { acuity (1) }\end{array}$ & 3 & VPS (3) & $\mathrm{NM} / 60$ & 3 & GBM (4) & High-grade (4) \\
\hline 4 & $\begin{array}{c}\text { Aboul-Enein } \\
\text { et al., } 2015 \\
(25)\end{array}$ & IV & $12(50 \%)$ & $50(24-67)$ & $\begin{array}{c}\text { Hemiparesis (4) } \\
\text { Diplopia (2) } \\
\text { Ataxia (2) } \\
\text { N/V (1) } \\
\text { CNIII palsy (1) }\end{array}$ & 3 & - & - & - & $\begin{array}{c}\text { Grade II } \\
\text { astrocytoma (1) } \\
\text { Glioma } \\
\text { (Unspecified) } \\
\text { (11) }\end{array}$ & $\begin{array}{l}\text { High-grade (3) } \\
\text { Low-grade (2) } \\
\text { Uncpecifed (7) }\end{array}$ \\
\hline 5 & $\begin{array}{c}\text { Sugita et al., } \\
2016 \text { (26) }\end{array}$ & IV & $2(50 \%)$ & $35(18-52)$ & $\begin{array}{c}\text { Headache }(2) \\
\text { Vertical gaze } \\
\text { palsy }(1)\end{array}$ & 2 & $\begin{array}{l}\text { VPS (1) } \\
\text { ETV (1) }\end{array}$ & - & - & Gliosarcoma (2) & High-grade (2) \\
\hline 6 & $\begin{array}{l}\text { Chernov } \\
\text { et al., } \\
2006(27)\end{array}$ & IV & $5(40 \%)$ & $35(20-71)$ & - & - & - & - & - & $\begin{array}{c}\text { Grade II } \\
\text { astrocytoma (2) } \\
\text { Anaplastic } \\
\text { astrocytoma (2) } \\
\text { ioma (Unspecified) }\end{array}$ & $\begin{array}{l}\text { High-grade (3) } \\
\text { Low-grade (2) } \\
\text { (1) }\end{array}$ \\
\hline 7 & $\begin{array}{c}\text { Amini et al., } \\
2006 \text { (28) }\end{array}$ & IV & $3(67 \%)$ & $43(40-52)$ & $\begin{array}{c}\text { Headache (3) } \\
\text { Decrease visual } \\
\text { acuity (2) } \\
\text { Diplopia (2) } \\
\text { N/V (2) } \\
\text { Decreased } \\
\text { LOC (2) }\end{array}$ & 3 & $\begin{array}{l}\text { VPS (1) } \\
\text { ETV (2) }\end{array}$ & & & GBM (3) & High-grade (3) \\
\hline 8 & $\begin{array}{c}\text { Barnett } \\
\text { et al., } \\
1995(29)\end{array}$ & IV & $2(50 \%)$ & 32 for both & $\begin{array}{l}\text { Headache (2) } \\
\text { Decrease visual } \\
\text { acuity (1) } \\
\text { N/V (1) } \\
\text { Dizziness (1) }\end{array}$ & 2 & VPS (2) & & & $\begin{array}{c}\text { Grade II } \\
\text { astrocytoma (2) }\end{array}$ & Low-grade (2) \\
\hline
\end{tabular}


Table I. Continued

\begin{tabular}{|c|c|c|c|c|c|c|c|c|c|c|c|}
\hline & \multirow{2}{*}{$\begin{array}{c}\text { Authors - } \\
\text { Year }\end{array}$} & \multirow{2}{*}{$\begin{array}{c}\text { Level } \\
\text { of } \\
\text { evidence }\end{array}$} & \multirow{2}{*}{$\begin{array}{c}\text { No. of } \\
\text { patients } \\
\text { (Male \%) }\end{array}$} & \multirow{2}{*}{$\begin{array}{c}\text { Age } \\
\text { median } \\
\text { (range) }\end{array}$} & \multirow{2}{*}{$\begin{array}{l}\text { Symptoms } \\
\text { (No. of } \\
\text { patients) }\end{array}$} & \multicolumn{2}{|c|}{$\mathrm{HCP}$} & \multirow{2}{*}{$\begin{array}{l}\text { Median } \\
\text { KPS } \\
\text { PreOp/ } \\
\text { PostOp }\end{array}$} & \multirow{2}{*}{$\begin{array}{c}\text { Median } \\
\text { tumor } \\
\text { size } \\
(\mathrm{cm})\end{array}$} & \multicolumn{2}{|c|}{ Histopathology } \\
\hline & & & & & & $\begin{array}{l}\text { No. } \\
\text { cases }\end{array}$ & $\begin{array}{c}\text { Modality of } \\
\text { shunting }\end{array}$ & & & Type (count) & WHO Grade \\
\hline 9 & $\begin{array}{c}\text { Dono et al., } \\
2020 \text { (30) }\end{array}$ & IV & $2(100 \%)$ & $39(24-54)$ & $\begin{array}{c}\text { Headache (2) } \\
\text { Ataxia (1) } \\
\text { N/V (1) } \\
\text { Decreased } \\
\text { LOC (1) }\end{array}$ & 2 & - & & & GBM (2) & High-grade (2) \\
\hline 10 & $\begin{array}{l}\text { Meyronet } \\
\text { et al., } \\
2017(21)\end{array}$ & IV & $6(0 \%)$ & $34(21-82)$ & - & - & - & - & - & $\begin{array}{c}\text { GBM (3) } \\
\text { Grade II } \\
\text { astrocytoma (2) } \\
\text { Pilocytic } \\
\text { astrocytoma (1) }\end{array}$ & $\begin{array}{l}\text { High-grade (3) } \\
\text { Low-grade (3) }\end{array}$ \\
\hline 11 & $\begin{array}{l}\text { D'Amico } \\
\text { et al., } \\
2018(22)\end{array}$ & IV & $8(88 \%)$ & $48(36-74)$ & - & 7 & $\begin{array}{l}\text { VPS (2) } \\
\operatorname{ETV~(5)~}\end{array}$ & - & - & GBM (8) & High-grade (8) \\
\hline 12 & $\begin{array}{l}\text { Choque- } \\
\text { Velasquez } \\
\text { et al., } \\
2020(15)^{*}\end{array}$ & IV & $13(77 \%)$ & $33(23-54)$ & $\begin{array}{c}\text { Acute or } \\
\text { progressive } \\
\text { hydrocephalus } \\
\text { related } \\
\text { symptoms (11) } \\
\text { Visual } \\
\text { impairment (7) } \\
\text { Memory } \\
\text { disturbances (4) } \\
\text { Motor deficits (2) }\end{array}$ & 13 & $\begin{array}{l}\text { VPS (5) } \\
\text { ETV (1) }\end{array}$ & - & 2.6 & $\begin{array}{c}\text { Pilocytic } \\
\text { astrocytoma (10) } \\
\text { Ependymoma (2) } \\
\text { Anaplastic } \\
\text { astrocytoma (2) } \\
\text { Grade II } \\
\text { astrocytoma (1) } \\
\text { GBM (2) }\end{array}$ & $\begin{array}{l}\text { High-grade (2) } \\
\text { Low-grade (15) }\end{array}$ \\
\hline
\end{tabular}

*This study includes 13 adults and 4 pediatric cases whose tumor type, grade and adjuvant treatment data were not distinguishable; all the cases were included in this table yet excluded from the pooled analysis. N/V, Nausea and Vomiting; CN, cranial nerve; LOC, level of consciousness; VPS, ventriculoperitoneal shunt; ETV, endoscopic third ventriculostomy; WHO, World Health Organization; NM, not mentioned.

minimal contrast enhancement. The median tumor size was $3.8 \mathrm{~cm}$, presenting comparable results to pineal region meningiomas $(15,32)$. This is likely due to the confined, small space of the pineal region, with an expansion of the tumor resulting in clinical symptoms. Of available data in our cohort, HGG represents $67.9 \%$, with glioblastoma being the most common type $(73.7 \%)$ of HGGs. This finding is consistent with the overall prevalence of $\mathrm{HGG}$ and glioblastoma subtype among the general CNS gliomas and perhaps explains the poor overall survival (35).

Treatment modalities and survival outcomes. We found that only $14.8 \%$ of patients had recurrence after initial treatment, while $70.6 \%$ experienced death due to disease progression. This is reflective of the low rate of GTR and the malignant nature of most cases leading to the high rate of death in our cohort. In contrast to our results, Yamaki et al. systematically reviewed papillary tumors affecting the pineal region and reported higher recurrence rates $(57 \%)$, yet recorded lower death rates (11\%) (36). This discrepancy reflects the differences in the biological behavior between gliomas and papillary tumors and further emphasizes the malignant nature of gliomas.

Our results imply a poor median OS in our cohort (12 months), which is attributed to the predominance of HGG (67.9\%); however, we find the wide survival range (2-78 months) reflective of the behavior variance among glioma subtypes. Additionally, we found a one-year OS rate of $60 \%$ among all PRGs, with glioblastoma showing the worst oneyear survival rate of 45 percent. In contrast, supratentorial glioma literature shows a better median overall survival (5.9 years) and a higher one-year survival rate (72-94\%), yet they reported a comparable glioblastoma one-year survival rate of $41 \%$ (3). These contrasting data support the role of the pineal region's anatomical complexity in worsening the survival outcome due to the proximity of critical structures in such a dense, deep space (37). Lastly, we found that LGG achieved a better survival rate than HGG $(p<0.01)$, in line with the general understanding of CNS glioma mortality (38). 
Table II. Overview of the management details of all included studies.

\begin{tabular}{|c|c|c|c|c|c|c|c|c|}
\hline \multirow{2}{*}{\multicolumn{2}{|c|}{$\begin{array}{l}\text { Authors - } \\
\text { Year }\end{array}$}} & \multicolumn{2}{|c|}{$\begin{array}{c}\text { Surgical } \\
\text { management }\end{array}$} & \multirow[t]{2}{*}{$\begin{array}{c}\text { Chemotherapy } \\
\text { No. patients }\end{array}$} & \multirow{2}{*}{$\begin{array}{l}\text { Radiotherapy } \\
\text { No. patients/ } \\
\text { median dose }\end{array}$} & \multirow[t]{2}{*}{$\begin{array}{l}\text { PFS median in } \\
\text { months (range) }\end{array}$} & \multirow[t]{2}{*}{$\begin{array}{l}\text { OS median in } \\
\text { months (range) }\end{array}$} & \multirow[t]{2}{*}{$\begin{array}{c}\text { Status } \\
\text { (No. patients) }\end{array}$} \\
\hline & & $\begin{array}{c}\text { Extent } \\
\text { of resection } \\
\text { (No. patients) }\end{array}$ & $\begin{array}{l}\text { Surgical } \\
\text { approach }\end{array}$ & & & & & \\
\hline 1 & $\begin{array}{l}\text { Niu et al., } \\
2020 \text { (23) }\end{array}$ & STR (3) & - & 2 & $2 / \mathrm{NM}$ & $12(10-16)$ & $12(10-16)$ & $\begin{array}{l}\text { A (1) } \\
\text { D (2) }\end{array}$ \\
\hline 2 & $\begin{array}{l}\text { Li et al., } \\
2019 \text { (4) }\end{array}$ & $\begin{array}{c}\text { GTR (8) } \\
\text { STR (12) } \\
\text { Biopsy (1) }\end{array}$ & $\begin{array}{l}\text { TT (11) } \\
\text { TCIH (7) } \\
\text { OTT (1) } \\
\text { SCIT (2) }\end{array}$ & 3 & $5 / \mathrm{NM}$ & $12(2-78)$ & $12(2-78)$ & $\begin{array}{l}\text { A (10) } \\
\text { D (11) }\end{array}$ \\
\hline 3 & $\begin{array}{l}\text { Orrego et al., } \\
2017 \text { (24) }\end{array}$ & $\begin{array}{l}\text { GTR (1) } \\
\text { STR (1) } \\
\text { PR (2) }\end{array}$ & $\begin{array}{l}\text { SCIT (2) } \\
\text { OCSO (1) } \\
\text { NM (1) }\end{array}$ & 2 & $4 / 5,800 \mathrm{cGy}$ & $7.5(6-9)$ & $20(6-32)$ & $\mathrm{D}(4)$ \\
\hline 4 & $\begin{array}{l}\text { Aboul-Enein } \\
\text { et al., } \\
2015(25)\end{array}$ & $\begin{array}{l}\text { GTR (9) } \\
\text { STR (3) }\end{array}$ & SCIT (12) & - & - & - & - & - \\
\hline 5 & $\begin{array}{l}\text { Sugita } \text { et al., } \\
2016(26)\end{array}$ & $\begin{array}{l}\text { GTR (1) } \\
\text { PR (1) }\end{array}$ & - & - & 2/4,000cGy & $7(9-11)$ & $18(13-24)$ & $\mathrm{D}(2)$ \\
\hline 6 & $\begin{array}{l}\text { Chernov et al., } \\
2006 \text { (27) }\end{array}$ & Biopsy (5) & - & 2 & $5 / \mathrm{NM}$ & - & - & - \\
\hline 7 & $\begin{array}{l}\text { Amini et al., } \\
2006 \text { (28) }\end{array}$ & $\begin{array}{c}\text { GTR (1) } \\
\text { PR (1) } \\
\text { Biopsy (1) }\end{array}$ & $\begin{array}{l}\text { SCIT (1) } \\
\text { TCSC (1) }\end{array}$ & 2 & $3 / 5,940 \mathrm{cGy}$ & - & $5(2-7)$ & D (3) \\
\hline 8 & $\begin{array}{l}\text { Barnett et al., } \\
1995 \text { (29) }\end{array}$ & $\begin{array}{l}\text { GTR (1) } \\
\text { PR (1) }\end{array}$ & SCIT (2) & & & - & $18(12-24)$ & A (2) \\
\hline 9 & $\begin{array}{l}\text { Dono et al., } \\
2020(30)\end{array}$ & $\begin{array}{c}\text { STR (1) } \\
\text { Biopsy (1) }\end{array}$ & - & 2 & 2/ NM & $9.6(7.6-11.7)$ & $15.5(12-19)$ & $\begin{array}{l}\text { D (1) } \\
\text { A (1) }\end{array}$ \\
\hline 10 & $\begin{array}{c}\text { Meyronet } \\
\text { et al., } 2017 \\
(21)\end{array}$ & Biopsy (6) & - & 5 & 3/ NM & $12(3-25)$ & $13(3-25)$ & $\mathrm{D}(6)$ \\
\hline 11 & $\begin{array}{c}\text { D'Amico } \\
\text { et al., } \\
2018(22)\end{array}$ & STR (8) & $\begin{array}{l}\text { SCIT (7) } \\
\text { TCIH (1) }\end{array}$ & 6 & 7/4,000cGy & $15(2-25)$ & $15(2-25)$ & $\begin{array}{l}\text { D (7) } \\
\text { A (1) }\end{array}$ \\
\hline 12 & $\begin{array}{c}\text { Choque- } \\
\text { Velasquez } \\
\text { et al., } \\
2020(15)^{*}\end{array}$ & $\begin{array}{l}\text { GTR (7) } \\
\text { STR (5) } \\
\text { PR (1) }\end{array}$ & $\begin{array}{l}\text { SCIT (12) } \\
\text { OTT (1) }\end{array}$ & 4 & $6 / 4,500 \mathrm{cGy}$ & & $83(52-148)$ & - \\
\hline
\end{tabular}

*This study includes 13 adults and 4 pediatric cases whose tumor type, grade and adjuvant treatment data were not distinguishable; all the cases were included in this table yet excluded from the pooled analysis. PFS, Progression free survival; OS, overall survival; GTR, gross total resection ( $>98 \%)$; STR, sub-total resection (90-98\%), PR, partial resection (<90\%); OTT, occipital transtentorial approach; SCSO, supracerebeloso suboccipital approach; TCSC transcortical/subchoroidal approach; TCIH, transcallosal interhemispheric approach; SCIT, supracerebellar infratentorial approach; TT, transcortical transventricular approach; cGy, centigray; A, alive; D, dead; NM, not mentioned.

Additionally, we found no difference in preoperative and postoperative KPS due to our scattered, scarce data; two studies reported KPS, containing only one case with postoperative data. Still, in one included article, Li et al. reported higher survival rates among LGG cases with KPS $>60$ than patients with KPS $<60(p=0.019)$ but failed to 
Table III. Summary of clinical characteristics, management strategies and outcomes of all pooled patients.

\begin{tabular}{|c|c|}
\hline Characteristics $(\mathrm{n}=81)$ & $\begin{array}{l}\text { Value (percentage } \\
\text { among available data) }\end{array}$ \\
\hline \multicolumn{2}{|l|}{ Demographics } \\
\hline Median age, Range $(\mathrm{N}=68)$ & $39,18-82$ Yrs. \\
\hline Gender $(\mathrm{N}=81)$ & Male: $44(54.3 \%)$ \\
\hline Most common presenting symptoms $(\mathrm{N}=65)$ & No. $(\%)$ \\
\hline Headache & $13(20.0 \%)$ \\
\hline Decreased visual acuity & $11(16.9 \%)$ \\
\hline Nausea and vomiting & $9(13.8 \%)$ \\
\hline Motor deficit & $7(10.8 \%)$ \\
\hline Diplopia & $7(10.8 \%)$ \\
\hline Ataxia & $6(9.2 \%)$ \\
\hline Memory impairment & $4(6.2 \%)$ \\
\hline Vertical gaze palsy & $3(4.6 \%)$ \\
\hline Decreased level of consciousness & $3(4.6 \%)$ \\
\hline Dizziness & $2(3.1 \%)$ \\
\hline Hydrocephalus $(\mathrm{N}=50)$ & No. $(\%)$ \\
\hline Ventriculoperitoneal shunt & $20(40.0 \%)$ \\
\hline Endoscopic third ventriculostomy & $12(24.0 \%)$ \\
\hline Not shunted & $18(36.0 \%)$ \\
\hline KPS & Median, Range \\
\hline Pre-operative $(\mathrm{N}=21)$ & $60,30-100$ \\
\hline Post-operative $(\mathrm{N}=1)$ & 60 \\
\hline Tumor size $(\mathrm{N}=27)$ & Median, Range \\
\hline Median & $3.8 \mathrm{~cm}$ \\
\hline Range & $2-6 \mathrm{~cm}$ \\
\hline Histopathological type/Grade $(\mathrm{N}=56)$ & No. $(\%)$ \\
\hline Glioblastoma IV & $28(50.0 \%)$ \\
\hline Astrocytoma II & $11(19.6 \%)$ \\
\hline Unspecified grade astrocytoma & $5(8.9 \%)$ \\
\hline Anaplastic astrocytoma III & $4(7.1 \%)$ \\
\hline Ependymoma II & $3(5.4 \%)$ \\
\hline Gliosarcoma IV & $2(3.6 \%)$ \\
\hline Pilocytic astrocytoma I & $1(1.8 \%)$ \\
\hline Ganglioglioma I & $1(1.8 \%)$ \\
\hline Central neurocytoma I & $1(1.8 \%)$ \\
\hline Surgery $(n=81)$ & No. $(\%)$ \\
\hline Gross-total resection ( $\geq 98 \%$ ) & $28(34.6 \%)$ \\
\hline Sub-total resection $(80-97 \%)$ & $33(40.7 \%)$ \\
\hline Partial resection $(<80 \%)$ & $7.4(7.4 \%)$ \\
\hline Biopsy & $14(17.3 \%)$ \\
\hline Surgical approach $(\mathrm{N}=61)$ & No. $(\%)$ \\
\hline Supracerebellar infratentorial approach & $38(62.3 \%)$ \\
\hline Transcortical transventricular approach & $11(18.0 \%)$ \\
\hline Transcallosal interhemispheric approach & $8(13.1 \%)$ \\
\hline Occipital transtentorial approach & $2(3.3 \%)$ \\
\hline Supracerebeloso suboccipital approach & $1(1.6 \%)$ \\
\hline Transcortical/Subchoroidal approach & $1(1.6 \%)$ \\
\hline Adjuvant therapy $(\mathrm{N}=53)$ & No. (\% among all cases) \\
\hline Chemotherapy & $26(32.1 \%)$ \\
\hline Radiotherapy & $33(40.7 \%)$ \\
\hline Median dose (Range) & $5,770 \mathrm{cGy}(1,800-10,080)$ \\
\hline Outcomes (Months) & Median (Range) \\
\hline Progression free survival $(\mathrm{N}=44)$ & $11.3(2-78)$ \\
\hline Overall survival $(\mathrm{N}=46)$ & $12.0(2-78)$ \\
\hline Recurrence & $12(14.8 \%)$ \\
\hline Status $(\mathrm{N}=51)$ & No. $(\%)$ \\
\hline Alive & $15(29.4 \%)$ \\
\hline Dead & $36(70.6 \%)$ \\
\hline
\end{tabular}

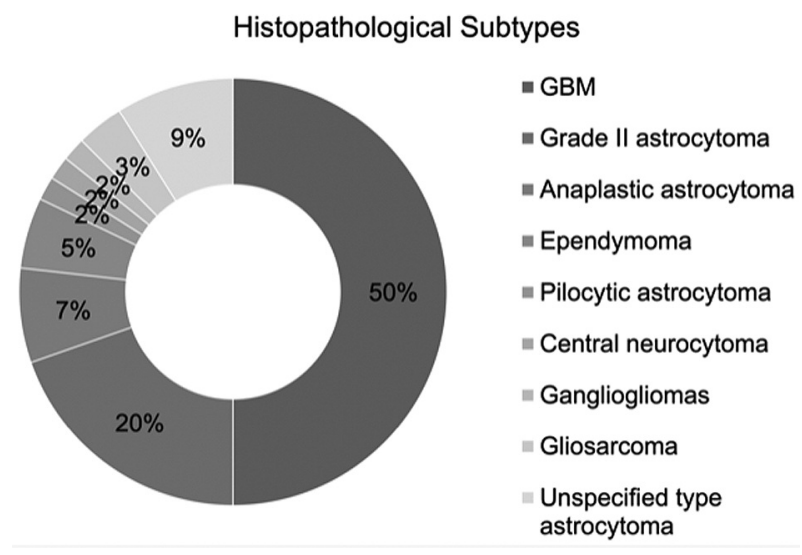

Figure 2. Pie chart demonstrating histopathological subtypes of included gliomas. GBM, Glioblastoma multiforme.

detect a significant difference in HGG $(p=0.077)$ (4). Regardless, several studies in the literature examined the KPS prognostic value on gliomas of different CNS regions and concluded that a high preoperative KPS score (>60-80) along with a prolonged pre-hospitalization duration of symptoms ( $\geq 2$ months) were associated with positive outcomes among glioma patients (39-41).

Data on the survival of shunting modalities among adults are scarce and controversial. Although most authors reported that ETV was more efficient despite its high failure rates, some argued that VPS is associated with prolonged survival and a better complication profile $(42,43)$. Interestingly, we found a superior survival benefit in patients who received VPS compared with cases who underwent ETV $(p<0.05)$.

Our results showed that the supracerebellar infratentorial approach was the preferred surgical corridor $(62.3 \%)$ as it provides direct access to the pineal region and third ventricle. This fundamentally offers a more uncomplicated dissection of the deep structures (25). In most cases, surgical resection failed to achieve GTR, documented in only $34.6 \%$ of our patients. These findings confirm the region's related surgical challenges and further emphasize the neurosurgeons' roles in navigating through available surgical approaches to achieve optimum tumor exposure and extent of resection.

In our review, 53 patients received adjuvant treatment; $32.1 \%$ received chemotherapy, and $40.7 \%$ received radiotherapy, with a median dose of 5,770 cGy. Although we did not find a survival correlation, adjuvant therapy has increased survival benefits in different cancers and has been a primary management element for different glioma subtypes (44-46). Therefore, avid research efforts, including clinical trials, have been employed to examine these therapeutic modalities $(47,48)$. 
a

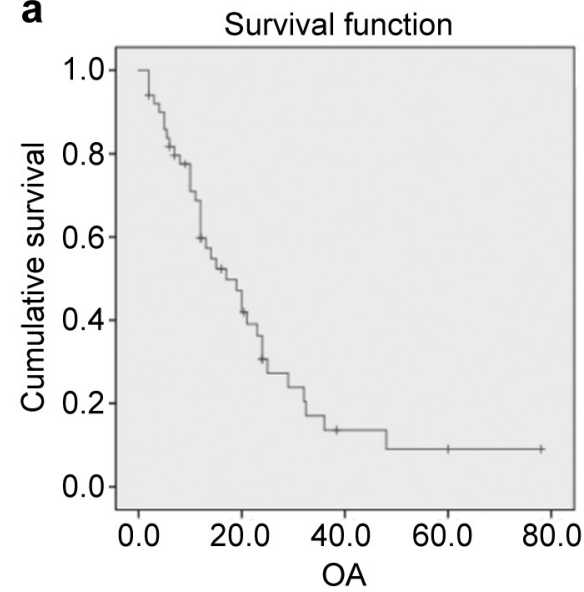

b

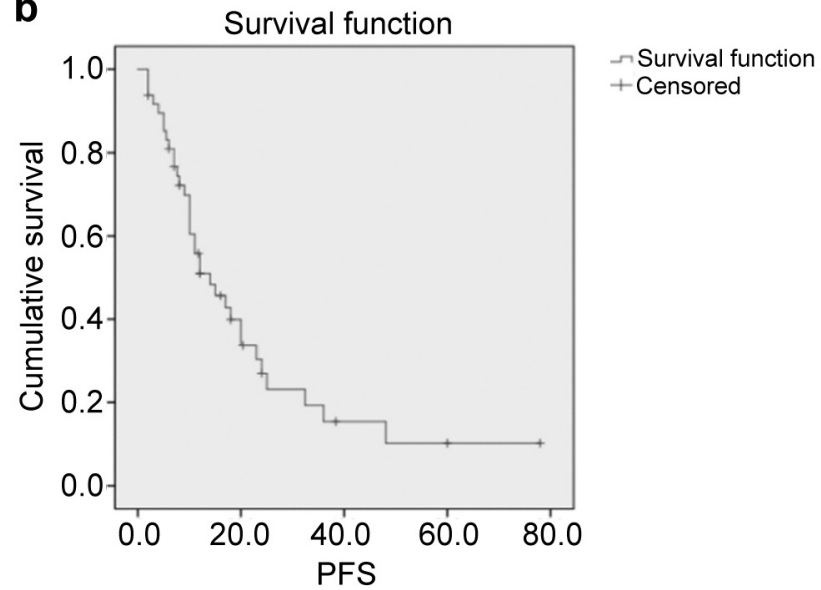

Figure 3. Kaplan-Meier survival curves for (a) OS $(n=44)$ and (b) PFS $(n=46)$ for the overall pooled cohort. OS, Overall survival; PFS, progression free survival.

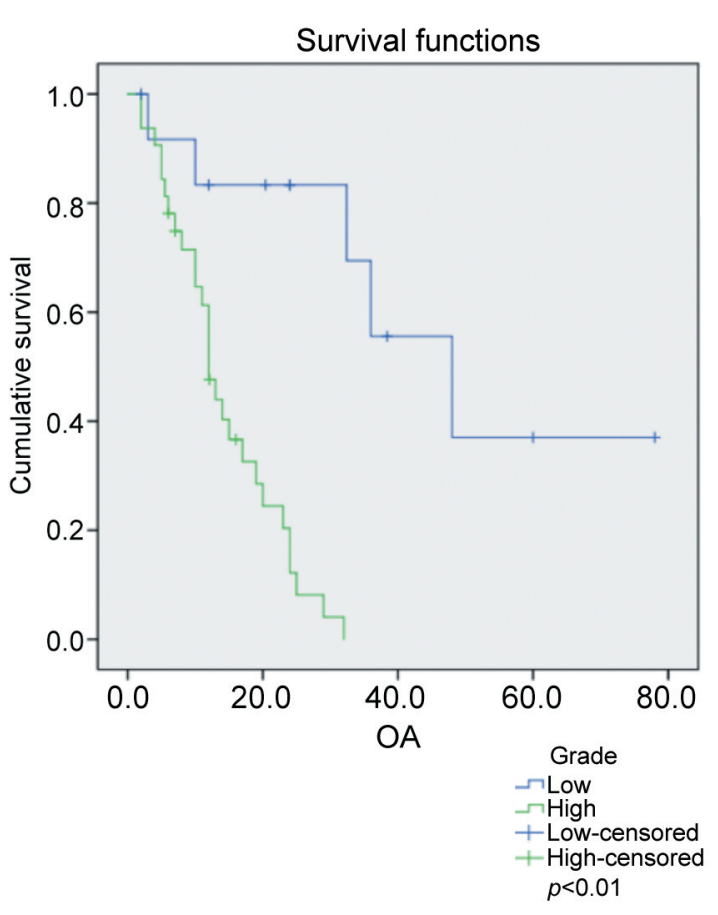

Figure 4. Kaplan-Meier survival curves comparing low-grade and highgrade gliomas (Log-rank test, $p<0.01$ ).

\section{Limitations}

This study has limitations. Our heterogeneous data and the small sample size challenged the statistical robustness, which further limited the pooled analysis. Although we detected significant survival differences, these results can be affected by confounding variables and potential bias. The inconsistency of study methodology and reported results of the included literature further limited our study findings.

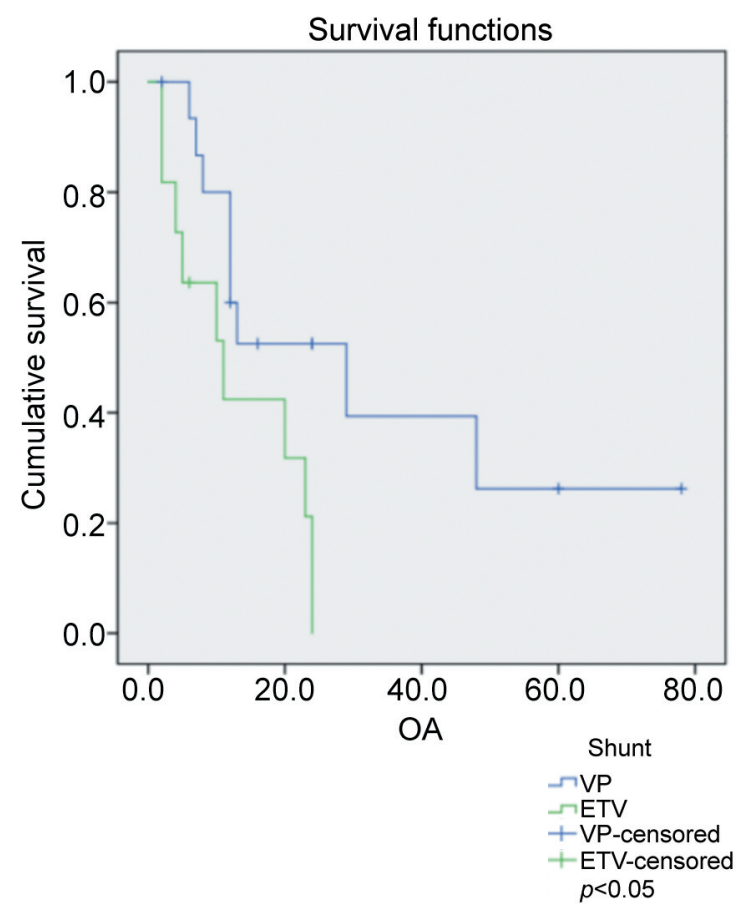

Figure 5. Kaplan-Meier survival curves comparing ventriculoperitoneal shunt (VPS) and endoscopic third ventriculostomy (ETV) (Log-rank test, $p<0.05$ ).

\section{Conclusion}

Gliomas are malignant neoplasms that can involve the pineal region and can be excessively challenging to manage. Our data support the overall poor prognosis of HGG compared to LGG. Although we found a survival benefit in employing VPS in contrast to ETV, we failed to detect a significant survival benefit based on the extent of resection or surgical approach. To optimize pineal region glioma 
outcomes, a personalized medicine approach integrating clinical and histomolecular data is necessary. Large, prospective studies are needed to establish a standard management strategy and provide reliable prognostic conclusions.

\section{Conflicts of Interest}

The Authors report no conflicts of interest concerning the materials or methods used in this study or the findings specified in this paper.

\section{Authors' Contributions}

Othman Bin Alamer: Conceptualization, Methodology, Data analysis, Writing - Original draft preparation; Navraj S. Sagoo, Scott E Rowe, and Aditya Dutta Gupta: Paper screening, Resources, Writing Reviewing and Editing; Paolo Palmisciano, Maryam Haider, Mohammed Alduhaymi and Ali S. Haider: Resources, Data extraction, and Writing - Reviewing and Editing; Tarek Y. El Ahmadieh, Kenny Yu, Aaron A. Cohen-Gado, and Ali S. Haider 1: Resources, Writing Critically reviewing the final draft.

\section{References}

1 Magrini S, Feletti A, Marton E and Longatti P: Gliomas of the pineal region. J Neurooncol 115(1): 103-111, 2013. PMID: 23820809. DOI: $10.1007 / \mathrm{s} 11060-013-1200-9$

2 Vaquero J, Ramiro J, Martínez R and Bravo G: Neurosurgical experience with tumours of the pineal region at Clinica Puerta de Hierro. Acta Neurochir (Wien) 116(1): 23-32, 1992. PMID: 1319669. DOI: $10.1007 / \mathrm{BF} 01541249$

3 Ostrom QT, Cote DJ, Ascha M, Kruchko C and Barnholtz-Sloan JS: Adult glioma incidence and survival by race or ethnicity in the United States from 2000 to 2014. JAMA Oncol 4(9): 1254-1262, 2018. PMID: 29931168. DOI: 10.1001/jamaoncol.2018.1789

4 Li D, Wen R, Gao Y, Xu Y, Xiong B, Gong F and Wang W: Pineal region gliomas: a single-center experience with 25 cases World Neurosurg 133: e6-e17, 2020. PMID: 31284062. DOI: 10.1016/j.wneu.2019.06.189

5 Stein BM: Supracerebellar-infratentorial approach to pineal tumors. Surg Neurol 11(5): 331-337, 1979. PMID: 441923.

6 Choque-Velasquez J, Resendiz-Nieves J, Jahromi BR, Colasanti R, Baluszek S, Muhammad S and Hernesniemi J: Midline and paramedian supracerebellar infratentorial approach to the pineal region: a comparative clinical study in 112 patients. World Neurosurg 137: e194-e207, 2020. PMID: 32001412. DOI: 10.1016/j.wneu.2020.01.137

7 Almahariq F, Raguz M, Romic D, Dlaka D, Oreskovic D, Sesar P and Chudy D: A biphasic tumor in posterior cranial fossa and the pineal region in young adult. Surg Neurol Int 11: 64, 2020. PMID: 32363059. DOI: $10.25259 /$ SNI_288_2019

8 Reis F, Faria AV, Zanardi VA, Menezes JR, Cendes F and Queiroz LS: Neuroimaging in pineal tumors. J Neuroimaging 16(1): 52-58, 2006. PMID: 16483277. DOI: 10.1177/1051228405001514

9 Quick-Weller J, Lescher S, Baumgarten P, Dinc N, Bruder M, Weise L, Seifert V and Marquardt G: Stereotactic biopsy of pineal lesions. World Neurosurg 96: 124-128, 2016. PMID: 27287513. DOI: 10.1016/j.wneu.2016.04.130
10 Little KM, Friedman AH and Fukushima T: Surgical approaches to pineal region tumors. J Neurooncol 54(3): 287-299, 2001. PMID: 11767294. DOI: 10.1023/a:1012766902431

11 Sood S, Hoeprich M and Ham SD: Pure endoscopic removal of pineal region tumors. Childs Nerv Syst 27(9): 1489-1492, 2011. PMID: 21607639. DOI: 10.1007/s00381-011-1490-1

12 Hernesniemi J, Romani R, Albayrak BS, Lehto H, Dashti R, Ramsey C 3rd, Karatas A, Cardia A, Navratil O, Piippo A, Fujiki M, Toninelli S and Niemelä M: Microsurgical management of pineal region lesions: personal experience with 119 patients. Surg Neurol 70(6): 576-583, 2008. PMID: 19055952. DOI: 10.1016/j.surneu.2008.07.019

13 Blakeley JO and Grossman SA: Management of pineal region tumors. Curr Treat Options Oncol 7(6): 505-516, 2006. PMID: 17032562. DOI: $10.1007 / \mathrm{s} 11864-006-0025-6$

14 Radovanovic I, Dizdarevic K, de Tribolet N, Masic T and Muminagic S: Pineal region tumors-neurosurgical review. Med Arh 63(3): 171-173, 2009. PMID: 20088167.

15 Choque-Velasquez J, Resendiz-Nieves J, Jahromi BR, Baluszek S, Muhammad S, Colasanti R and Hernesniemi J: Long-term survival outcomes of pineal region gliomas. J Neurooncol 148(3): 651-658, 2020. PMID: 32613272. DOI: 10.1007/s11060-020-03571-z

16 Choque-Velasquez J, Resendiz-Nieves J, Jahromi BR, Colasanti R, Raj R, Vehviläinen J, Tynninen $\mathrm{O}$, Collan J, Niemelä M and Hernesniemi J: Extent of resection and long-term survival of pineal region tumors in helsinki neurosurgery. World Neurosurg 131: e379-e391, 2019. PMID: 31369883. DOI: 10.1016/j.wneu. 2019.07.169

17 Stowe HB, Miller CR, Wu J, Randazzo DM and Ju AW: Pineal region glioblastoma, a case report and literature review. Front Oncol 7: 123, 2017. PMID: 28660172. DOI: 10.3389/fonc.2017.00123

18 Page MJ, McKenzie JE, Bossuyt PM, Boutron I, Hoffmann TC, Mulrow CD, Shamseer L, Tetzlaff JM, Akl EA, Brennan SE, Chou R, Glanville J, Grimshaw JM, Hróbjartsson A, Lalu MM, Li T, Loder EW, Mayo-Wilson E, McDonald S, McGuinness LA, Stewart LA, Thomas J, Tricco AC, Welch VA, Whiting P and Moher D: The PRISMA 2020 statement: an updated guideline for reporting systematic reviews. BMJ 372: n71, 2021. PMID: 33782057. DOI: 10.1136/bmj.n71

19 The Joanna Briggs Institute Critical Appraisal tools for use in JBI Systematic Reviews. Checklist for Case Series. Available at: http://joannabriggs.org/research/critical-appraisal-tools.html [Last accessed on January 26, 2022]

20 Howick J, Chalmers I, Glasziou P, Greenhalgh T, Heneghan C, Liberati A and Hodgkinson M: The Oxford 2011 Levels of Evidence. Oxford Centre Evidence-Based Medicine, 2011.

21 Meyronet D, Esteban-Mader M, Bonnet C, Joly MO, Uro-Coste E, Amiel-Benouaich A, Forest F, Rousselot-Denis C, BurelVandenbos F, Bourg V, Guyotat J, Fenouil T, Jouvet A, Honnorat $\mathrm{J}$ and Ducray F: Characteristics of H3 K27M-mutant gliomas in adults. Neuro Oncol 19(8): 1127-1134, 2017. PMID: 28201752. DOI: $10.1093 /$ neuonc/now274

22 D'Amico RS, Zanazzi G, Wu P, Canoll P and Bruce JN: Pineal region glioblastomas display features of diffuse midline and nonmidline gliomas. J Neurooncol 140(1): 63-73, 2018. PMID: 29992434. DOI: 10.1007/s11060-018-2931-4

23 Niu X, Wang C, Zhou X, Yang Y, Liu Y, Zhang Y and Mao Q: Pineal region glioblastomas: Clinical characteristics, treatment, and survival outcome. World Neurosurg 146: e799-e810, 2021. PMID: 33186787. DOI: 10.1016/j.wneu.2020.11.016 
24 Orrego E, Casavilca S, Garcia-Corrochano P, Rojas-Meza S, Castillo $\mathrm{M}$ and Castaneda CA: Glioblastoma of pineal region: report of four cases and literature review. CNS Oncol 6(4): 251-259, 2017. PMID: 28990813. DOI: 10.2217/cns-2016-0047

25 Aboul-Enein H, El-Aziz Sabry AA and Hafez Farhoud A: Supracerebellar infratentorial approach with paramedian expansion for posterior third ventricular and pineal region lesions. Clin Neurol Neurosurg 139: 100-109, 2015. PMID: 26426425. DOI: 10.1016/j.clineuro.2015.08.009

26 Sugita Y, Terasaki M, Tanigawa K, Ohshima K, Morioka M, Higaki K, Nakagawa S, Shimokawa $S$ and Nakashima S: Gliosarcomas arising from the pineal gland region: uncommon localization and rare tumors. Neuropathology 36(1): 56-63, 2016. PMID: 26183264. DOI: 10.1111/neup.12226

27 Chernov MF, Kamikawa S, Yamane F, Ishihara S, Kubo O and Hori T: Neurofiberscopic biopsy of tumors of the pineal region and posterior third ventricle: indications, technique, complications, and results. Neurosurgery 59(2): 267-77; discussion 267-77, 2006. PMID: 16883167. DOI: 10.1227/01.NEU.0000223504.29243.0B

28 Amini A, Schmidt RH, Salzman KL, Chin SS and Couldwell WT: Glioblastoma multiforme of the pineal region. J Neurooncol 79(3): 307-314, 2006. PMID: 16645719. DOI: 10.1007/s11060-006-9145-x

29 Barnett DW, Olson JJ, Thomas WG and Hunter SB: Low-grade astrocytomas arising from the pineal gland. Surg Neurol 43(1): 705; discussion 75-6, 1995. PMID: 7701429. DOI: 10.1016/ 0090-3019(95)80043-g

30 Dono A, Takayasu T, Ballester LY and Esquenazi Y: Adult diffuse midline gliomas: Clinical, radiological, and genetic characteristics. J Clin Neurosci 82(Pt A): 1-8, 2020. PMID: 33317715. DOI: 10.1016/j.jocn.2020.10.005

31 Abay EO 2nd, Laws ER Jr, Grado GL, Bruckman JE, Forbes GS, Gomez MR and Scott M: Pineal tumors in children and adolescents. Treatment by CSF shunting and radiotherapy. J Neurosurg 55(6): 889-895, 1981. PMID: 7299463. DOI: 10.3171/jns.1981.55.6.0889

32 Konovalov AN, Spallone A and Pitzkhelauri DI: Meningioma of the pineal region: a surgical series of 10 cases. J Neurosurg 85(4): 586-590, 1996. PMID: 8814160. DOI: 10.3171/jns.1996.85.4.0586

33 Konovalov AN and Pitskhelauri DI: Principles of treatment of the pineal region tumors. Surg Neurol 59(4): 250-268, 2003. PMID: 12748006. DOI: 10.1016/s0090-3019(03)00080-6

34 Zhang Z, Wang H, Cheng H, Fan Y, Hang C, Sun K and Zhu L: Management of hydrocephalus secondary to pineal region tumors. Clin Neurol Neurosurg 115(9): 1809-1813, 2013. PMID: 23743211. DOI: 10.1016/j.clineuro.2013.05.009

35 Ostrom QT, Patil N, Cioffi G, Waite K, Kruchko C and BarnholtzSloan JS: CBTRUS statistical report: Primary brain and other central nervous system tumors diagnosed in the United States in 2013-2017. Neuro Oncol 22(12 Suppl 2): iv1-iv96, 2020. PMID: 33123732. DOI: 10.1093/neuonc/noaa200

36 Yamaki VN, Solla DJF, Ribeiro RR, da Silva SA, Teixeira MJ and Figueiredo EG: Papillary tumor of the pineal region: Systematic review and analysis of prognostic factors. Neurosurgery 85(3): E420-E429, 2019. PMID: 30989225. DOI: 10.1093/neuros/nyz062

37 Hervey-Jumper SL and Berger MS: Maximizing safe resection of low- and high-grade glioma. J Neurooncol 130(2): 269-282, 2016. PMID: 27174197. DOI: 10.1007/s11060-016-2110-4

38 Lapointe S, Perry A and Butowski NA: Primary brain tumours in adults. Lancet 392(10145): 432-446, 2018. PMID: 30060998. DOI: $10.1016 / \mathrm{S} 0140-6736(18) 30990-5$
39 Zhang P, Wang X, Ji N, Xie J, Han J, Ren X, Song G, Wu R, Zhang $\mathrm{L}$ and Gao Z: Clinical, radiological, and pathological features of 33 adult unilateral thalamic gliomas. World J Surg Oncol 14: 78, 2016. PMID: 26965578. DOI: 10.1186/s12957-016-0820-x

40 Cao L, Li C, Zhang Y and Gui S: Surgical resection of unilateral thalamic tumors in adults: approaches and outcomes. BMC Neurol 15: 229, 2015. PMID: 26545867. DOI: 10.1186/s12883-015-0487-x

41 Rasmussen BK, Hansen S, Laursen RJ, Kosteljanetz M, Schultz H, Nørgård BM, Guldberg R and Gradel KO: Epidemiology of glioma: clinical characteristics, symptoms, and predictors of glioma patients grade I-IV in the the Danish Neuro-Oncology Registry. J Neurooncol 135(3): 571-579, 2017. PMID: 28861666. DOI: 10.1007/s11060-017-2607-5

42 Gonda DD, Kim TE, Warnke PC, Kasper EM, Carter BS and Chen CC: Ventriculoperitoneal shunting versus endoscopic third ventriculostomy in the treatment of patients with hydrocephalus related to metastasis. Surg Neurol Int 3: 97, 2012. PMID: 23061013. DOI: 10.4103/2152-7806.100185

43 Gholampour S, Bahmani $\mathrm{M}$ and Shariati A: Comparing the efficiency of two treatment methods of hydrocephalus: Shunt implantation and endoscopic third ventriculostomy. Basic Clin Neurosci 10(3): 185-198, 2019. PMID: 31462974. DOI: 10.32598/bcn.9.10.285

44 Xu S, Tang L, Li X, Fan F and Liu Z: Immunotherapy for glioma: Current management and future application. Cancer Lett 476: 112, 2020. PMID: 32044356. DOI: 10.1016/j.canlet.2020.02.002

45 Wang TJC and Mehta MP: Low-grade glioma radiotherapy treatment and trials. Neurosurg Clin N Am 30(1): 111-118, 2019. PMID: 30470398. DOI: 10.1016/j.nec.2018.08.008

46 Weller M, van den Bent M, Tonn JC, Stupp R, Preusser M, CohenJonathan-Moyal E, Henriksson R, Le Rhun E, Balana C, Chinot O, Bendszus M, Reijneveld JC, Dhermain F, French P, Marosi C, Watts C, Oberg I, Pilkington G, Baumert BG, Taphoorn MJB, Hegi M, Westphal M, Reifenberger G, Soffietti R, Wick W and European Association for Neuro-Oncology (EANO) Task Force on Gliomas: European Association for Neuro-Oncology (EANO) guideline on the diagnosis and treatment of adult astrocytic and oligodendroglial gliomas. Lancet Oncol 18(6): e315-e329, 2017. PMID: 28483413. DOI: 10.1016/S1470-2045(17)30194-8

47 Stupp R, Mason WP, van den Bent MJ, Weller M, Fisher B, Taphoorn MJ, Belanger K, Brandes AA, Marosi C, Bogdahn U, Curschmann J, Janzer RC, Ludwin SK, Gorlia T, Allgeier A, Lacombe D, Cairncross JG, Eisenhauer E, Mirimanoff RO, European Organisation for Research and Treatment of Cancer Brain Tumor and Radiotherapy Groups and National Cancer Institute of Canada Clinical Trials Group: Radiotherapy plus concomitant and adjuvant temozolomide for glioblastoma. N Engl J Med 352(10): 987-996, 2005. PMID: 15758009. DOI: 10.1056/NEJMoa043330

48 Tom MC, Cahill DP, Buckner JC, Dietrich J, Parsons MW and Yu JS: Management for different glioma subtypes: Are all low-grade gliomas created equal? Am Soc Clin Oncol Educ Book 39: 133145, 2019. PMID: 31099638. DOI: 10.1200/EDBK_238353

Received December 20, 2021

Revised January 22, 2022

Accepted January 26, 2022 